\title{
Correction to: Implications of the Overlapping Degree Between Proximal Fibula and Tibia for Placing the Optimal Syndesmotic Screw: A Virtual Cadaveric Study
}

\author{
Gu-Hee Jung ${ }^{1,2} \cdot$ Jun-Young Lee ${ }^{3} \cdot$ Jae-Hwan Lim ${ }^{4} \cdot$ Hyeon-Joon Lee ${ }^{3} \cdot J^{\prime}$ Jue-Yeon Lee ${ }^{3}$
}

Published online: 6 July 2021

(c) Indian Orthopaedics Association 2021

\section{Correction to: Indian Journal of Orthopaedics (2021) https://doi.org/10.1007/s43465-021-00437-y}

The original version of this article, published on June 15, 2021, contained a mistake. The funding note was missing. The correct information is given below. The original article has been corrected.

Funding note.

This work was supported by a grant from the Clinical Medicine Research Institute of the Chosun University Hospital (2019).

The original article can be found online at https://doi.org/10.1007/ s43465-021-00437-y.

Jun-Young Lee

leejy88@chosun.ac.kr

Gu-Hee Jung

jyujin2001@ hotmail.com

Jae-Hwan Lim

eternul@naver.com

Hyeon-Joon Lee

dtscream@naver.com

June-Yeon Lee

republickor7@naver.com

1 Department of Orthopaedic Surgery, Gyeongsang National University, College of Medicine, Gyeongsang National University Changwon Hospital, 11, Samjeongja-ro, Seongsan-gu, Changwon-si 51472, Korea
2 College of Medicine, Medical ICT Convergence Research Center, Institute of Health Sciences, Gyeongsang National University, 816 Beongil 15, Jinju-daero, Jinju-si 52727, Korea

3 Department of Orthopaedic Surgery, Chosun University, Chosun University Hospital, 365 Pilmundae-ro, Dong-gu, Gwangju 61453, Korea

4 Department of Orthopaedic Surgery, Gwangju Suwan Hospital, 370 Imbangueldae-ro, Gangsan-gu, Gwangju 62247, Korea 